\title{
Major neurocognitive disorder and academic achievement: a case report
}

\begin{abstract}
Neurocognitive disorders are identified by a significant decline in relation to prior levels of cognitive functioning. This case report highlights a 47year-old Caucasian female who has a history of sub-occipital craniotomy for the removal of a grade II brain stem astrocytoma at age 17 and a difficult childhood. Of interest is this patient's ability to overcome Major Neurocognitive Disorder while achieving advanced academic success. Recent neuropsychological evaluation and specific deficit findings are discussed in regards to this patient continually achieving academic success during cognition deficits. Common factors of neurocognitive decline are well documented but consistent integration of these factors such as age, gender, education, diagnostics, treatment, and therapy options are lacking in literature. This case highlights the importance of identifying all factors involved in neurocognitive decline and, as importantly, those factors involved in overcoming the issues related to this diagnosis. Concluded was that timely and accurate diagnosis, location of tumor, which treatment options are utilized, and post treatment monitoring can each have an effect on patient outcome.
\end{abstract}

Volume 6 Issue 3 - 2016

\author{
Nancy A Bergquist,' Bailey A Weidner, ${ }^{2}$ Diana \\ Finley,' Theresa M Meloche,' Tony L Brown³ \\ 'Major General Hugh G Robinson Center for Neuropsychiatry, \\ USA \\ ${ }^{2}$ University of Colorado, Colorado Springs, CO, USA \\ ${ }^{3}$ Department of Neurology, Columbia College of Physicians and \\ Surgeons, USA
}

Correspondence: Tony Brown, Columbia University, USA, Email tbrown@post.harvard.edu

Received: March 27, 2016 | Published: July 07, 2016

Keywords: neurocognitive disorder, astrocytoma, neurosurgery, academia, brain

\section{Introduction}

Compared with brain tumors in adults, a much higher percentage of pediatric brain tumors arise in the posterior fossa, which includes the brain stem and cerebellum. While astrocytoma's can be found elsewhere in the brain, the majority are found in the posterior fossa. These infratentorial astrocytoma tumors comprise as many as two thirds of all pediatric brain tumors. ${ }^{1}$ An astrocytoma is typically a childhood tumor and is of glial cell origin. These glioma tumors start in the brain or spinal cord tissue and do not spread beyond these defining margins or tend to affect other organs. While signs and symptoms of a brain tumor can vary depending on tumor location, generally, headaches, seizures, memory loss, and behavioral changes make up the most common signs and symptoms in the presence of an astrocytoma. Most symptomatic children have endured several visits to a physician before the correct diagnosis has been made, and often they are misdiagnosed with gastrointestinal disorders. Once an astrocytoma has been identified, initial treatment is usually neurosurgical resection. Of interest is this patient's ability to overcome Major Neurocognitive Disorder (MND) while achieving advanced level academic success.

\section{Astroycytoma in major neurocognitive disorder}

The patient noted in this case study underwent pre-surgical imaging to identify the size and precise location of the tumor, and then posterior surgical invasion of the cranium during surgery to access the brain stem. No post-surgical chemotherapy or radiation therapy was performed. Complete tumor removal is the goal of surgery and a biopsy of tumor material is often performed during surgery as well. Further treatment of chemotherapy is reserved for tumors that progress despite surgery. Personalized chemotherapy is being pursued now that there is better understanding of the molecular components of this tumor. Radiotherapy has generally not been recommended, but stereotactic radiotherapy and conformal proton beam radiotherapy may be reasonable options in the setting of relapse or progression.". ${ }^{2}$ Reducing post-treatment neurocognitive deficits in these tumor patients is of utmost concern. Pre- and postsurgical neuropsychological testing is often performed on the patient. Posterior fossa resection surgery often leaves neurocognitive deficits, ${ }^{3,4}$ however, controversy exists as to the degree these deficits are implicated as sequelae of surgery. With identified deficits noted, statistically significant relationships between both the preoperative and post-operative deficits were identified. ${ }^{5}$ The presence of oculomotor impairment was associated with both verbal fluency deficits and imagery disorders. A significant correlation between the presence of ataxia, and its subcategory, dysmetria, existed with attention dysfunction and planning dysfunction. They wrote, "After surgery no patient showed a worsening of his/her cognitive profile; the relationships between clinical, intraoperative, and radiological findings were substantially confirmed.". ${ }^{5}$ The neurocognitive deficits noted in this case report exist 30years after the surgical intervention, are without any prior contrasting neuropsychological testing results, and are affected by several factors.

\section{Case presentation}

Patient history included a difficult upbringing with personal alcohol abuse starting at age ten years, and ending ten years ago. She related that her mother had told her she had received psychotherapy between the ages of ten to twelve years, but she does not recall the therapy. She attended group therapy for alcohol addiction for ten years until recently when she quit participation due to new educational demands. Medical history includes bilateral polyps requiring surgery over several years, bilateral deafness requiring corrective surgery over several years, a cochlear implant that did improve her hearing, and a failed surgery to improve her vision. She had a turbulent relationship with her mother who had a history of bipolar disorder that was a dual diagnosis with her alcohol and prescription medication abuse. The patient's mother recently died of a drug overdose and had been non-adherent with her breast cancer treatment regimen. This patient related that a good relationship existed with her father, until his death from a myocardial infarction when she was 18years of age. She is 
currently divorced and related unstable intimate relationships since her divorce. She related no academic problems during her elementary years, but reported conduct problems during her middle school years.

After undergoing neurosurgical resection of her brain tumor, she dropped out of high school and ultimately earned her GED. She went on to earn a Bachelor of Science degree in biology, and subsequently graduated and became licensed as a Doctor of Chiropractic. Her current educational endeavor is also clinically focused, a Doctor of Medicine. She related that she has pushed hard and was able to struggle through her various and progressive educational programs, and that she had to work hard for her academic achievements. Recent neuropsychological evaluation results revealed compromised attention/concentration abilities under time constraints, as well as learning and memory with immediate and delayed memory recall deficiencies. This patient also encountered difficulty with organization, item misplacement, daydreaming, difficulty with sustained attention, and difficulty staying alert in situations where she felt bored, as well as shallow and fleeting relationships. She was noted to have a strong sense of self-worth and a positive self-evaluation. She has typically been intolerant of inactivity and relays a possible penchant for momentary excitement, fleeting adventures, and shortsighted hedonism. She also related an insatiable search for stimulation, affection, and a rejection of traditional gender roles

\section{Exacerbating factors of MND}

Factors in this case such as her female gender, length of time since her surgery, long-term alcohol abuse, chaotic childhood, challenges with bilateral deafness, and multiple surgeries for other issues each, and collectively have contributed to neurocognitive decline. Alcohol use has been well documented to affect the brain and result in impaired memory recall, slowed reaction times, and speech difficulties. ${ }^{6}$ Longterm alcohol abuse has been generally recognized as a causative factor in brain atrophy from neuronal death. This patent abused alcohol for 27 years, and begins the abuse during her developmental years, at age 10. Other sequelae of long -term alcohol abuse include permanent memory loss, as noted in Korsakoff dementia, liver disease and cancer, nutritional deficiencies, and ataxia. Certain medications are known to affect cognition, as well. This patient's current respiratory medications (Symbicort, Pulmicort, and Proventil), though, have not been associated with neurocognitive decline. IQ testing is a common method used to document neurocognitive decline. While testing was indicated given this patient's history, no IQ testing was ever performed. "The rate of IQ decline is associated with several risk factors, including younger age at time of treatment, longer time since treatment, female sex, as well as clinical variables such as hydrocephalus, use of radiotherapy and radiotherapy dose, and the volume of the brain that received treatment." ${ }^{4}$ Regarding additional factors, the authors stated, "Chaotic and dysfunctional family environments have a substantial adverse effect on neurocognitive recovery from traumatic brain injury in school-age children." ${ }^{4} \mathrm{~A}$ proper diagnosis of MND's must include impairment in one or more of the following cognitive domains: Learning and memory, complex attention, social cognition, executive ability, language, or perceptual motor abilities. ${ }^{7}$ Recent neuropsychological evaluation findings suggested this patient has Major Neurocognitive Disorder due to a medical condition (brainstem astrocytoma with neurosurgical resection). Results from this recent evaluation revealed impairment across several cognitive domains. There was quantifiable outcome assessment to compare these results to, although perhaps, the patient's lack of recall regarding academic problems in elementary school could be utilized as a historical comparison. Quantitative literature analysis regarding the magnitude of neurocognitive deficits specifically in pediatric brain stem tumor survivors was recently conducted. Late effects of neurocognitive decline were noted to be moderate to severe across broad neurocognitive domains, for survivors of posterior fossa tumors, ${ }^{8}$ as was also the case for this patient. Her academic success, though, are contradictory to the statement that "Disruptions in cognitive development are among the most frequently observed late effects in this group of survivors, and include possible long-term disruptions in brain development, cognitive function, and later school and work performance". ${ }^{8}$

\section{Study strategies for MND patients}

Methods of improving neurocognitive function are fairly well documented in the literature, but offer little insight as to the reasons behind this patient's significant academic success despite her ongoing cognition issues. Cognitive remediation treatment has historically involved several forms of therapy, such as cognitive behavioral therapy and memory improvement techniques. Evidence is lacking in regard to known academic success improvement strategies. One study noted that while training with a memory notebook helped increase memory, correlated was only a slightly increased academic success. ${ }^{9}$ For the current patient, she reported utilization of the following:

a. One-on-one tutoring for five hours per week.

b. Remote webinar instruction. Nine hours per week of new material. She repeated each 90-minute session twice during the week for a total of 27 hours of additional instruction per week.

c. Medical Student Review Course. This coursework helps the learner effectively integrate basic science concepts in preparation for medical board examinations.

Along with the methods that this patient utilized, other suggestions to improve academic success in patients with MND include:

i. Study group participation to aid in the retention of novel material.

ii. Verbal and visual imagery mnemonic aids to help retain information. Today there are many online sources available in many fields of study that animate, rather than condense, material for greater visual and auditory effects on memory.

iii. Repetitive use of practice question banks, class-specific learning videos, and flash cards to aid in creating long term memory.

iv. The use of memory aids in daily life such as a planner, calendar, to-do list, and pocket recorder.

v. Avoidance of cluttered workspaces and background noise that reduce visual and auditory stress.

vi. Online cognitive training programs that improve speed of recognition and recall.

Although she did not take advantage of them, this patient was deemed eligible for academic accommodations based on her diagnosed impairment and MND. Diagnosed patients should consider the benefits of receiving these testing accommodations:

a. Extra allotted time to complete tests.

b. A quiet room in order to minimize distractions related to her decreased processing speed and attentional challenges.

Seating placement at the front of the class to help with attention and concentration. 


\section{Discussion}

Difficulty in qualifying neurocognitive decline in this case arises from an absence of prior comparative testing. Even if pre-surgical testing had been performed, testing while experiencing deficits from a diagnosed brain tumor that also presents with functional impairment would likely have been abnormal, and thus, would not have been indicative of any truly normal function that the patient had enjoyed prior to any neurological insults. If pre-surgical testing is performed on these patients and compared to each one's own post-surgical testing, the variables of abnormal pre-surgical data compared to postsurgical data poses a problem in attaining accurate comparisons. Lack of any pre- or post-surgical testing until years after surgery, as in this case, also poses a problem in qualifying the neurocognitive results. Regular post-surgical testing is recommended for pediatric brain tumor patients. In the case of this patient no testing had been performed in prior 30years that had passed since her surgery.

\section{Conclusion}

A myriad of contributing factors related to the neurocognitive decline and the lack of comparative and objective data in this case has created difficulty in the accurate assignment of blame regarding her deficits. Recent neuropsychological testing attributed her deficits to her brain tumor and subsequent tumor resection, despite the lack of prior testing with which to make a comparison. Greater understanding of the clinical presentation of these tumors and judicious use of modern neuro imaging techniques may lead to more efficacious therapies and the potential for less post-treatment deficits. This patient had a strong drive to avoid female gender roles, which may have contributed to her educational pursuits in previously male dominated professions. Due to a strong sense of self, she has been able to navigate challenges until she has succeeded in her educational goals. No comprehensive data exists that has an explanation for this patient's neurocognitive decline with concurrent advanced academic achievements.

Increased interest exists in the reduction of neurocognitive effects in brain tumor patients through improved treatment options. The establishment of baseline neurocognitive testing at an early age should become mainstream, in order to identify early cognitive deficits and to later provide a comparative point if the need arises. Understanding the multiple factors that contribute to neurocognitive decline as well as the factors that contribute to improvement are important for overall patient success. Social and societal implications exist regarding the well-being of neurocognitive disorder patients. Further research is needed to understand the impact of strong personal factors such as self-will and self-esteem, contribute to the ability to achieve advanced academic success, while the patient still experiences many factors that contribute to neurocognitive decline.

\section{Consent}

Written informed consent was obtained from the patient for publication of this case report. A copy of the written consent is available for review by the Editor-in-Chief of this journal.

\section{Authors' contributions}

NB analyzed the patient history and data. All authors researched the literature and provided analysis and scholarly sources cited in this paper, contributed in writing the manuscript, and read and approved the final manuscript. TB is managing author.

\section{Acknowledgments}

None.

\section{Conflicts of interest}

Author declares there are no conflicts of interest.

\section{Funding}

None.

\section{References}

1. Tabatabaei SM. Posterior Fossa Tumor in Children. Iranian Journal of Child Neurology. 2012;6(2):19-24

2. Fehling MG, Craciunas SC. Editorial: High-grade intramedullary astrocytomas: What is the best surgical option? J Neurosurg Spine. 2010;12(2):141-142.

3. Gehrke AK, Baisley MC, Sonck AL, et al. Neurocognitive deficits following primary brain tumor treatment: Systematic review of a decade of comparative studies. J Neurooncol. 2013;115(2):135-142.

4. Mulher RK, Merchant TE, Gajjar A, et al. Late neurocognitive sequelae in survivors of brain tumours in childhood. Lancet Oncology. 2004;5(7):399-408.

5. Di Rocco C. Preoperative and postoperative neurological, neuropsychological and behavioral impairment in children with posterior cranial fossa astrocytomas and medulloblastomas: The role of the tumor and the impact of the surgical treatment. Childs Nerv Syst. 2010;26(9):1173-1188.

6. National Institute on Alcohol Abuse and Alcoholism. Alcohol's Damaging Effects on the Brain. National Institute on Alcohol Abuse and Alcoholism. 2004.

7. Schnyer DM. Major and Mild Neurocognitive Disroders. SMSherman (Author), Encyclopedia of Mental Health ( 2nd Edn) Elsevier. 2016. p. 33-38.

8. Robinson KE, Fraley CE, Pearson MM, et al. Neurocognitive late effects of pediatric brain tumors of the posterior fossa: A quantitative review. J Int Neuropsychol Soc. 2013; 19(1): 44-53.

9. Kerns KA, Thomson J. Case study: implementation of a compensatory memory system in a school age child with severe memory impairment. Pediatr Rehabil. 1998;2(2):77-87. 